\title{
Gastroesophageal Reflux Disease after Bariatric Surgery. A New Way for the Treatment and Prevention
}

Keywords: Bariatric surgery; Gastroesophageal reflux disease: Modification of surgical procedures

\begin{abstract}
Background: The number of bariatric procedures is constantly on the rise, and with them an increase in the number of "side effects" that are directly related to the technical characteristics of their performance. Among these is the formation of gastroesophageal reflux disease (GERD) and frequently hiatus hernia $(\mathrm{HH})$, in which the use of known types of fundoplication seems impossible.

Methods: We analyzed the results of treatment of 864 patients: Lap sleeve gastrectomy (LSG)-522 (60.4\%), Lap gastric bypass (LGB)$342(39.6 \%)$. In 464 (53.7\%) patients initially diagnosed with GERD, 183 (21.1\%) cases were combined with $\mathrm{HH}$. In the 1st group $(287,32.6 \%)$ standard methods were used. In the 2nd group $(577,66.7 \%)$ there was a formation of an anti-reflux valve (ARV). In the 1st group, 148 (51.5\%) showed signs of GERD. In the 2nd group, 316 (54.7\%) showed signs of GERD.

Results: Regardless of the type of operation, GERD symptoms appeared in $63(45.3 \%)$ patients in the 1st group and $28(10.7 \%)$ in the 2nd group. In the 1st group, the clinical picture of 121 (81.7\%) patients with GERD worsened; in the 2nd group, on the contrary, signs of GERD offset were seen in 280 (88.6\%) patients.

Conclusions: The typical methods for LSG, LGB, and Lap biliopancreatic diversion/doudenal switch (LBPD-DS) operations form the prerequisites for the development of GERD, and the applied prevention/treatment methods have low efficacy. Modification of operations with the formation of the ARV reduces the chance of developing GERD and can be recommended for widespread use.
\end{abstract}

\section{Introduction}

The World Health Organization (WHO) declared obesity an epidemic of the $21^{\text {st }}$ century. According to WHO, about $30 \%$ of the world's population (more than 2 billion people) are considered overweight $[1,2]$. Obesity, atrial hypertension $(\mathrm{AH})$, diabetes mellitus type 2 (DM 2), and dyslipoproteinemia are the main components of metabolic syndrome (MS), which is the leading risk factor for cardiovascular disease. Complications of MS cause a high mortality rate among working-age population [3].

The high efficiency of surgical treatment of MS is well known, and its usage has become routine. The number of bariatric operations continues to progressively increase from year to year. Their efficacy regarding the treatment of the basic components of MS has been proven [4-6]. We decided to focus on two popular procedures-sleeve gastrectomy (LSG) and gastric bypass (LGB). In our clinic, these operations have been widely used since 2007. These operations are the "gold standard" for treatment of the basic components of MS in many countries, including Russia. The likelihood of developing gastro-esophageal reflux disease (GERD) after these procedures is greatly increased and if it is present, the disease is aggravated. After
Journal of

Obesity and

Bariatrics

\section{Michael B. Fishman ${ }^{1}$, Zvi H. Perry ${ }^{2}$ and Leonid Lantsberg ${ }^{2}$}

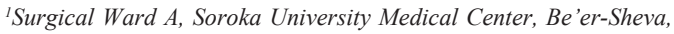
Israel

${ }^{2}$ Department of Faculty Surgery, First Pavlov State Medical University of St. Petersburg, Research Institute of Surgery and Emergency Medicine, Russia

\section{*Address for Correspondence}

Zvi H. Perry, Surgical Ward A, Soroka University Medical Center, PO Box 151, Be'er-Sheva - 64101, Israel, Tel: +972-50-340-0902/+972-8-6400610; Fax: +972-8-647-7633; E-mail: zperry@bgu.ac.il

Submission: 18 October, 2017

Accepted: 27 November, 2017

Published: : 06 December, 2017

Copyright: @ 2017 Fishman MB, et al. This is an open access article distributed under the Creative Commons Attribution License, which permits unrestricted use, distribution, and reproduction in any medium, provided the original work is properly cited.

bariatric surgery (such as LSG) GERD symptoms occur in 30-50\% of patients [7]. In these cases, the mechanisms of GERD have not yet been well understood. The fact remains that an LSG operation, and to a lesser extent LGB, leads to the development or aggravation of GERD. This pattern is harmful for the quality of life of our patients. In these operations, the weakening of the esophageal peristalsis results in a reduction of esophageal clearance. It reduces the contractile ability of the esophagus wall, weakening the pressure in the lower esophageal sphincter (LES). This leads to the de-structuring antireflux function of the LES, and slowing of gastric emptying [8]. These effects are the result of dissection of ligaments, viscera, the parietal peritoneum, and the lower esophageal-diaphragmatic membrane (continuing as a separate structure in the posterior mediastinum). As a result of these manipulations the angle of His is destroyed [9]. A partial destruction of the muscle mechanisms reduces LES tone. The most persistent change is accompanied by partial damage to the muscle tissue at the time of the LES manipulation. It normally runs along a fold of mucous membrane (plica cardiaca)-inner bottom border, and ends at the cardia of the diaphragm, from the left surface of the abdominal part of the esophagus. This leads to the relaxation of LES, and a pressure reduction. This forms a single space between the stump of the stomach and esophagus. In addition, after LSG and LGB, the fundus of the stomach stump is missing (after LGB isolated), and the place where the air bubble is usually localized is missing. Normally, the intra-abdominal pressure of the air bubble should help make a tight fit of the mucous membrane of the valve to the right wall of the abdominal part of the esophagus. In the absence of a gas bubble, the gas content of the gastric stump falls freely into the esophagus. Also, as a result of the operation, the residual stomach develops gastric paresis. The resulting days after surgery show peristalsis that is asynchronous, which leads to disruption of motor function. Aggressive substances are not cleared from the esophagus properly, which in turn leads to the development (or worsening) of inflammatory changes in the mucous. This further stimulates existing 
disorders of contractile ability which develop into a "vicious cycle". It creates the conditions that lead to the development of GERD, manifestations of which arise from the first days after the operation.

Surgical treatment of GERD involves fundoplication in a variety of ways $[10,11]$. However, the application of known methods of fundoplication in the treatment/prevention of GERD after bariatric surgery is not possible due to their technical features.

Thus, the absence of effective methods of prevention/treatment of this pathology is the unsolved problem of modern bariatric surgery. In the current study, we introduce our modification for the treatment of $\mathrm{HH}$ and an anti-reflux treatment for bariatric procedure patients.

\section{Methods and Materials}

Of all the patients operated on between 2007 and 2016 we formed a group consisting of 864 cases. These patients performed laparoscopic bariatric surgery (LSG and LGB), including VIKING 3D and Da Vinci technology. In $628(72.6 \%)$ cases, the patients were operated with the diagnosis of MS, the remaining 236 (27.3\%) underwent surgery for obesity of varying degrees; 563 were women $(65.1 \%)$, with a mean age of $41.34 \pm 9.24$ years; 301 (34.8\%) were men, with a mean age of $39.9 \pm 6.88$ years. BMI averaged $49 \pm 22.5 \mathrm{~kg} / \mathrm{m}^{2}$. LSG operation was performed in $522(60.4 \%)$ patients; in $342(65.5 \%)$ cases the operation was performed in the original modification to form an anti-reflux valve (ARV) and in $180(34.5 \%)$ cases the operation was performed by standard methods. LGB operation was performed in 342 (39.5\%) of the patients.

Among all operated patients, 464 (53.7\%) initially had GERD, which in $183(21.1 \%)$ cases was combined with HH. GERD was defined as a feeling of heartburn that is engulfing most of the day, in more than 3 days of the week, similar to the results seen in the QOL in reflux questionnaire [12]. All patients were divided into two groups. The $1^{\text {st }}$ group included 287 (32.6\%) cases (operations carried out by standard methods) and the $2^{\text {nd }}$ group, 577 (66.7\%) cases (operations by the developed technique for the formation of an ARV). In addition, each group was divided into two subgroups, with GERD symptoms and without them. Accordingly, in the $1^{\text {st }}$ group, 148 (51.5\%) cases had initial signs of GERD and in 139 (48.4\%) these were absent. In the $2^{\text {nd }}$ group, $316(54.7 \%)$ initially had symptoms of GERD and 261 (45.2\%) did not.

We performed routine studies of the study algorithm. This modification of operations to form the ARV further described by the example LSG operation. On this same principle, formation of the ARV was carried out during the operation LGB and LBPD-DS. LGB was performed with Lontron modification: alimentary loop (AP) $150 \mathrm{~cm}$, biliopancreatic loop (BPP) $75 \mathrm{~cm}$. The results obtained were subjected to mathematical and statistical analysis in the dynamics, with a maximum term of 7 years of observations.

Due to the fact we did not interview, change diagnosis or treatment plan for our patients, and all that was done was accumulating their data from medical records, the project was exempt from IRB.

Sample size was computed using the WINPEPI computer program (http://www.brixtonhealth.com/pepi4windows.html), using the COMPARE function (simple proportions study) with the following assumptions: Odds Ratio of 2 or less was considered negligible, power was set at $80 \%$, and $\alpha=0.05$. The proportion assumed as baseline was $30 \%$, and a ratio of 1:1 was defined. With these assumptions, the minimal sample size needed is 141 in each group or 282 in total. After continuity correction, the minimal number needed was set at 306 (153 in each group).

\section{Statistical analysis}

We analyzed the data using descriptive and analytical statistics: independent samples $t$ test, one-way ANOVA and $\chi 2$.

Statistical significance was considered when $\mathrm{p}<0.05$

\section{Procedure}

To perform the operation with the formation of the modified $\mathrm{ARV}$, we had the patient in the supine position. The surgeon was working between the legs of the patient. The $1^{\text {st }}$ assistant was situated at the right of the patient, with the $2^{\text {nd }}$ on his left. Monitors were installed behind the patient's head, on his right and left. Trocars were placed the same as when performing a standard LSG operation. Placing trocars is standard, taking into account the individual patient. Usually we provide access to the abdomen through four trocars, two $13 \mathrm{~mm}$ trocars (for the stapler, tools) and two $10 \mathrm{~mm}$ trocars (laparoscope, liver retractor). In case of need we use five $(5 \mathrm{~mm}$ ) trocars when applicable. Mobilization on the greater curvature of the stomach is performed by the usual method, $2 \mathrm{~cm}$ proximal from the pylorus and to the left of the diaphragmatic legs. Then we perform a dissection of the ligaments, parietal and visceral peritoneum, the lower esophageal diaphragmatic membrane to the anterior surface to the left diaphragmatic cruse.

These structures are cut in front of the left diaphragmatic cruses, thus avoiding injury of the esophagus and posterior vagus nerve. These manipulations should be well visualized, anterior to the diaphragmatic surface of the left crus and the left side surface of the abdominal esophagus, with its direct link to the stomach.

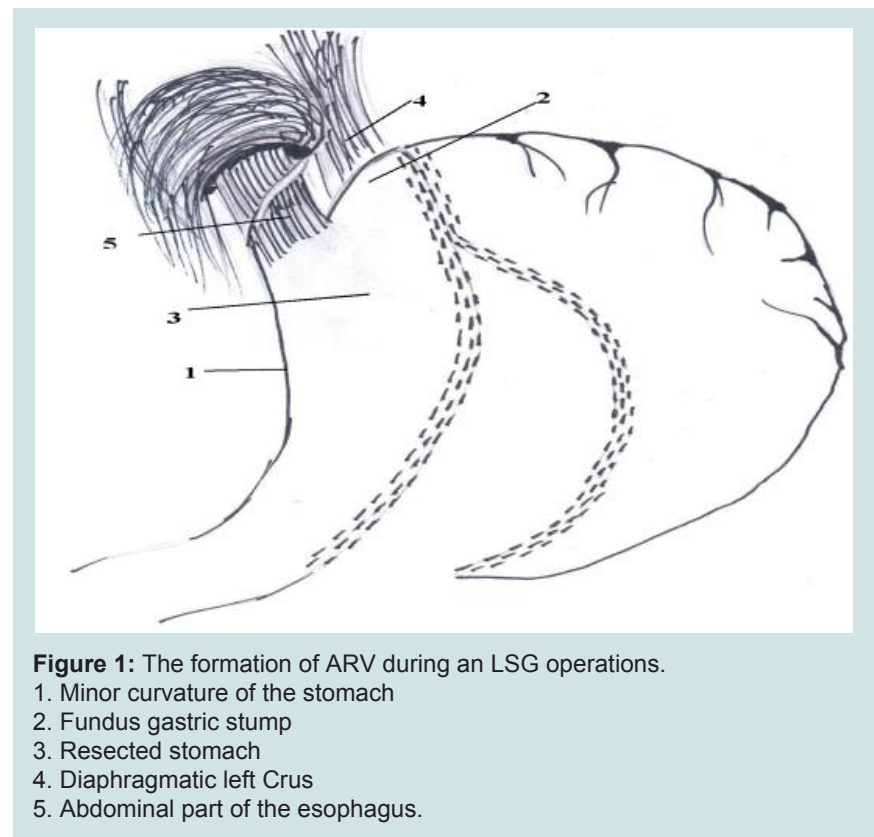


Citation: Fishman MB, Perry ZH, Lantsberg L. Gastroesophageal Reflux Disease after Bariatric Surgery. A New Way for the Treatment and Prevention. J Obes Bariatrics. 2017;4(1): 5.

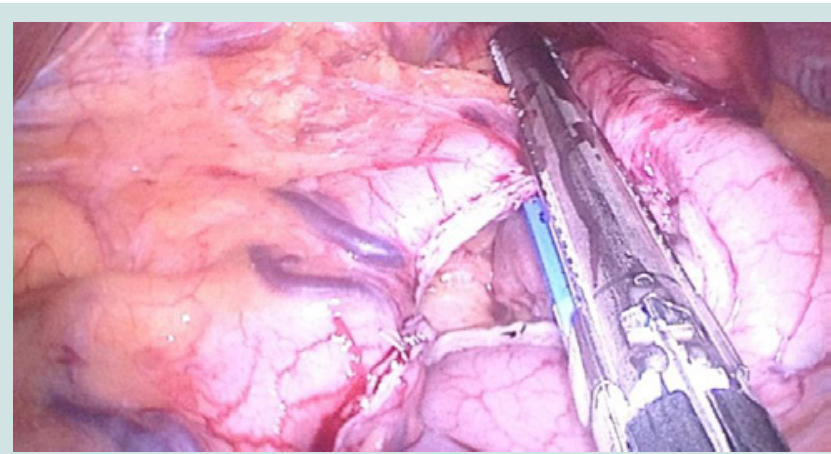

Figure 2: Intraoperative picture of gastrectomy in the formation of the ARV (2-3 cm left from Hiss angle).

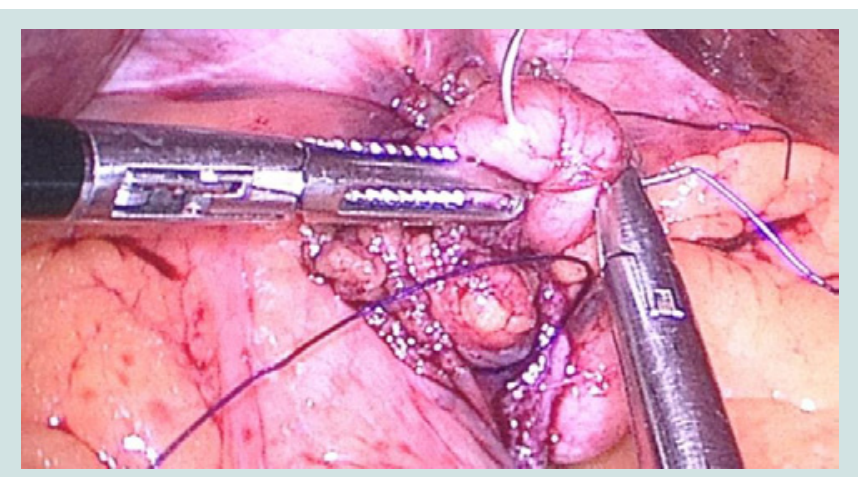

Figure 3: Intraoperative picture of the partial reconstruction of the fundus of the stomach stump in the formation of ARV.

Then, we do the gastrectomy on the width of the gauge nasogastric tube. In this case, the resection line is shifted to $2-3 \mathrm{~cm}$ to the left corner of branch block (Figures 1 and 2).

We carry out a resection of the stomach with the preserved part of its medial surface. We visualize the angle of His and impose a single node joint that secures the "bottom" of the stomach and the left crus of the diaphragmatic and abdominal part of the esophagus (Figures 3-5).

The composition of this should include the previously dissected peritoneum and diaphragmatic-esophageal membrane. Continuous suture, starting as high as possible, re-captured by the staple line seam, left diaphragmatic crus, abdominal part of the esophagus, and the previous (nodal) suture. Then continuous suture moves distally, with immersion peritonization of all the suture line staples (Figure 6).

In cases where LSG is performed in the presence of $\mathrm{HH}$ surgical technique is slightly different. Initially, in the peritoneal cavity one should extract the hernia contents (Figure 7). The stomach is mobilized on the greater curvature. In addition, tissue dissection is performed as is done in Nissen fundoplication.

This completes the mobilization of the phrenic-esophageal region. Such dissection should be carried out along the phrenic crus, reducing the likelihood of damage to the surrounding tissue. The result should be clearly visualized seeing the angle of Hiss, diaphragmatic crus, and abdominal esophagus. After the above procedure is performed we continue with the gastrectomy. The resected portion of the stomach is moved into the container below. As a result, in the epigastric area one has released space and this facilitates further manipulations. Running suturing of diaphragmatic crus (cruroraphia) is then performed (Figure 8). If necessary, this cruroraphia is performed in conjunction with a mesh implant. Imposing the nodal joints requires that the peritoneum and diaphragmatic-esophageal membrane are included in their composition. This cruroraphia shifts the abdominal part of the esophagus to the left and front. Less frequently, one might need a cruroraphiain front and if necessary it may be performed additionally. Next, formed ARV and peritonization suture line are performed by the above procedure. In addition to the first suture captured more diaphragmatic right crus (Figure 4). As a result, this run back cruroraphia partially recreated the bottom of the resected stomach, of Hiss and gastro-esophageal junction is secure fixed intraabdominally.

The above-described method of the original formation of the ARV for the prevention/treatment of GERD/HH can be applied after all bariatric operations (LGB and LBPD-DS).

\section{Results}

Studies have shown that when the procedure changes anatomical

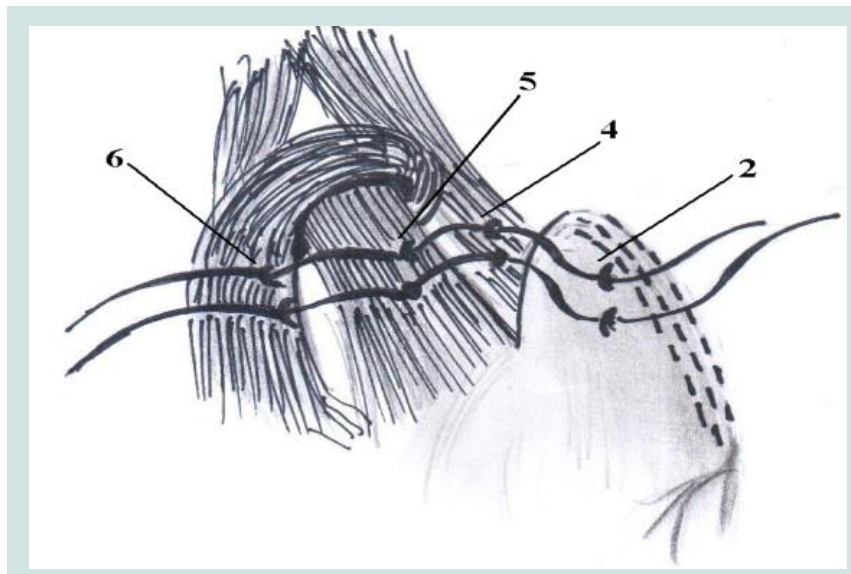

Figure 4: Suturing in the partial reconstruction of the fundus, the formation of the ARV and the Hiss acute angle.

2. Fundus gastric stump.

4,6. Diaphragmatic left and right Crus

5. Abdominal part of the esophagus.

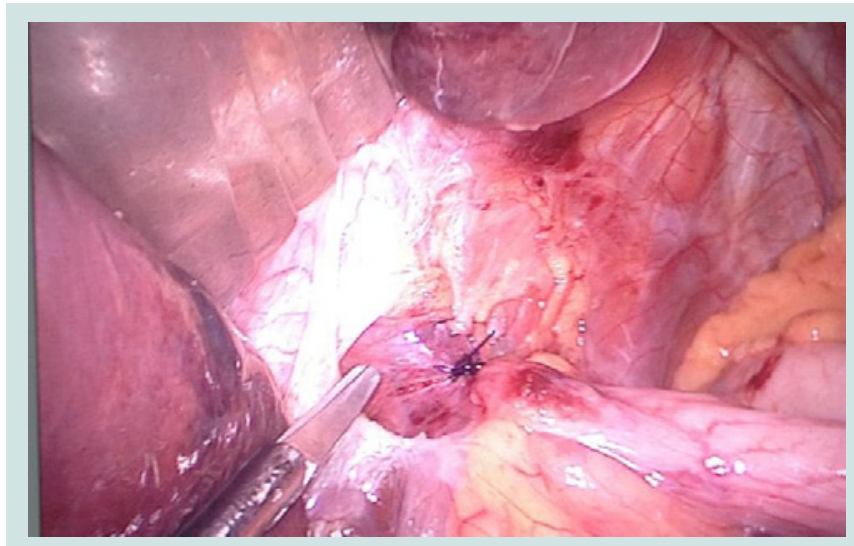

Figure 5: Intraoperative picture fixing tissues in the Hiss angle. Recreate the fundus of the stomach left aside. 
Citation: Fishman MB, Perry ZH, Lantsberg L. Gastroesophageal Reflux Disease after Bariatric Surgery. A New Way for the Treatment and Prevention. J Obes Bariatrics. 2017;4(1): 5.

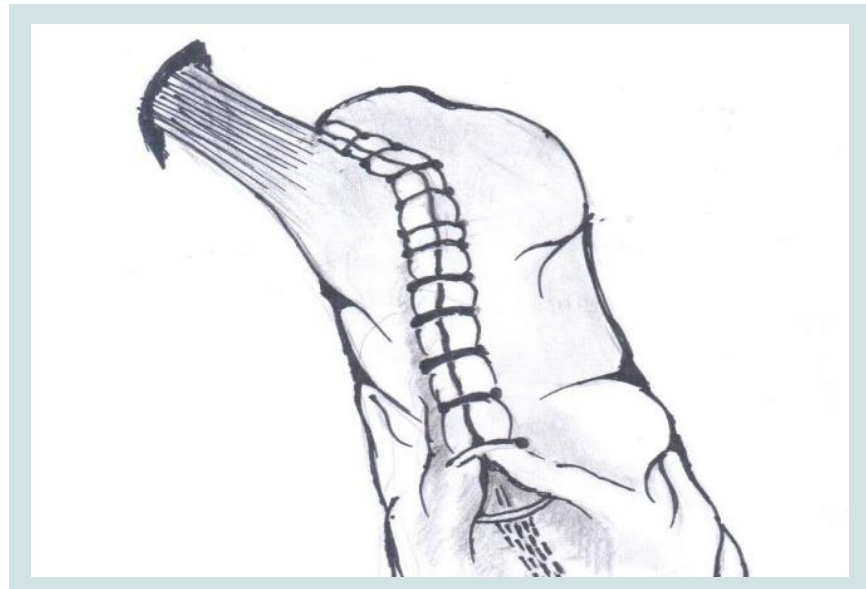

Figure 6: Formed fundus of the stomach and dive continuous suture line staples.

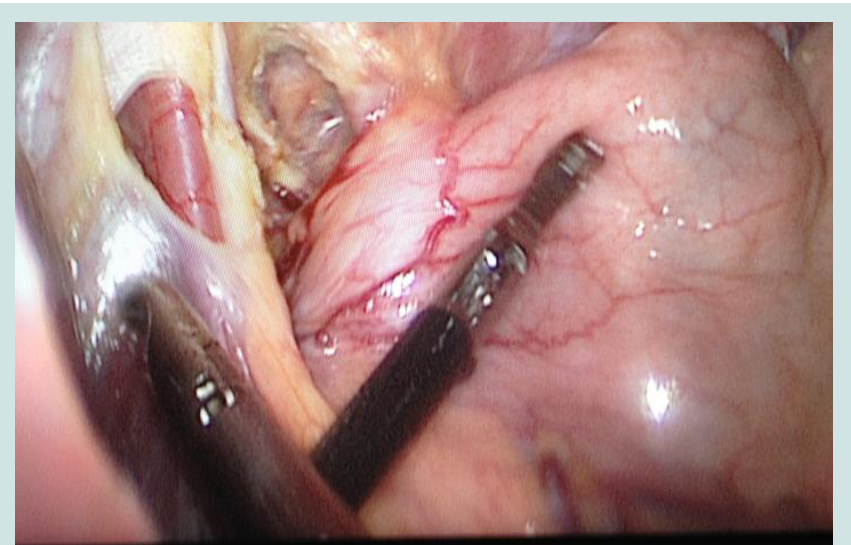

Figure 7: Intraoperative picture extraction from the mediastinum of the stomach into the abdominal cavity at $\mathrm{HH}$.

structures, which play an important role in the formation of anti-reflux mechanisms, GERD develops. Thus, anatomical and physiological balance in the gastro-esophageal area is crucial in bariatric procedures. They create the conditions that can lead to the development of GERD. When the initial presence of GERD is before surgery, the disease just gets worse.

As a result, regardless of the type of operation, when looking in patients at the 1st group (control), from the first day after surgery and during the entire observation period, GERD symptoms appeared in $63(45.3 \%)$ patients who were operated. In the $2^{\text {nd }}$ group of patients (cases with ARV) symptoms of GERD occurred in 28 (10.7\%) patients $(\mathrm{p}<0.05)$. In the $1^{\text {st }}$ group (control), 121 patients $(81.7 \%)$ have shown a clinical picture of GERD symptoms getting worse. In contrast, in the $2^{\text {nd }}$ group (cases), in 280 patients $(88.6 \%)$ GERD symptoms were minimized $(\mathrm{p}<0.001)$.

The modified operation with the formation of the ARV has a number of advantages and is clinically justified. With minimal additional intervention we were able to accomplish a mechanism that reduces the likelihood of GERD in the postoperative period. It is a relatively safe and easy method (after mastering the technique) to reduce the factors contributing to the development of GERD.
This method allows us to increase the effectiveness of bariatric surgery and to reduce the development of adverse effects and complications. It is characterized by good tolerability of the modified operations followed by rapid recovery.

Thus, the use in bariatric practice of the proposed modification to the formation of ARV allows us to generate an environment conducive to the prevention/treatment of GERD, $\mathrm{HH}$, and increase their efficiency.

\section{Discussion}

The surgical treatment of GERD, mainly caused by $\mathrm{HH}$, means performance of fundoplication in accordance with existing principles and types of operations used [8]. However, after bariatric operations, as a result of the technical features of these operations the use of some methods of fundoplication is virtually impossible. Typically, in cases where initially there were known GERD symptoms or a known $\mathrm{HH}$, cruroraphia is usually performed, but it might not be effective in the future.

As a result of the proposed modification to the formation of ARV, normal anatomical position, and function of the cardia, anti-reflux mechanisms are partially restored. This occurs as a result of the partial restoration of the integrity of the peritoneum, the high-pressure zone in the distal esophagus, intra-abdominal position (crus at the level of the diaphragm) abdominal esophagus, LES, recreating an acute His angle branch block. The formed fundus of the stomach stump retains an air bubble, contributing to a more tightly fit mucous valve. There is a speedier recovery of the esophageal peristalsis, and as a consequence increased esophageal clearance, an increase in pressure in the LES, and accelerated emptying of the newly formed stomach.

All this allows preventing and treating the GERD that is caused or aggravated by the bariatric procedure. It reduces the number of patients with GERD, as seen by our results. In addition, the ARV reduced the likelihood of developing gastric stump wall defect in the Hiss angle (the most frequently occurring complications of surgery LSG).

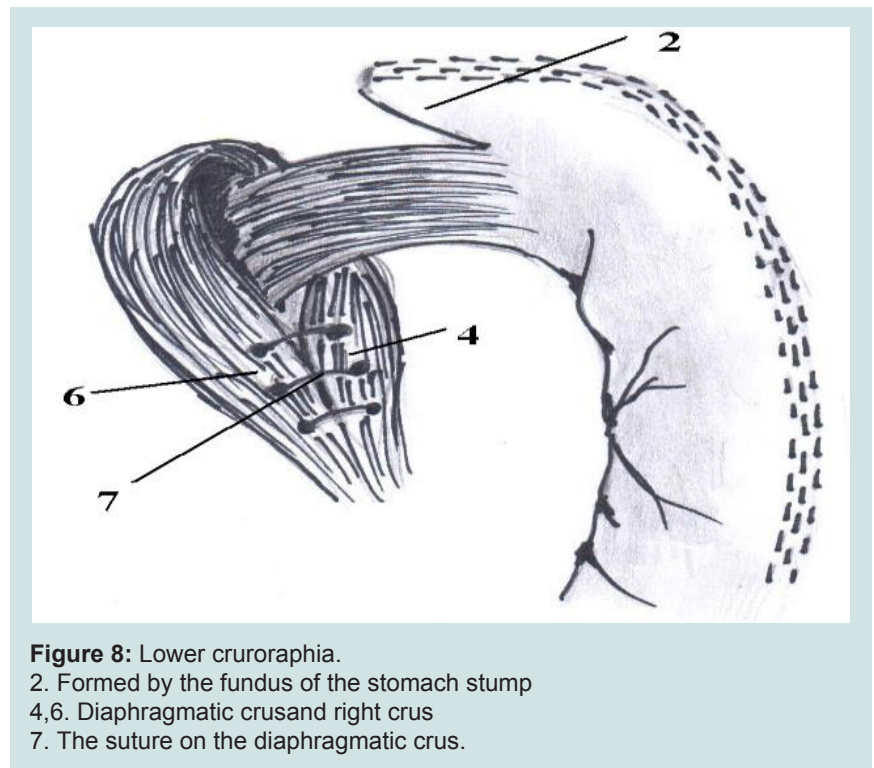


Citation: Fishman MB, Perry ZH, Lantsberg L. Gastroesophageal Reflux Disease after Bariatric Surgery. A New Way for the Treatment and Prevention. J Obes Bariatrics. 2017;4(1): 5.

ISSN: $2377-9284$

\section{Conclusion}

LSG, LRYGB, LBPD-DS operations all form the prerequisites for development of GERD, and the applied prevention/treatment methods have low efficiency.

Modified operation with the formation of the ARV is physiologically justified, can improve results, and may be recommended for wider use.

\section{References}

1. Vandevijvere S, Chow CC, Hall KD, Umali E, Swinburn BA (2015) Increased food energy supply as a major driver of the obesity epidemic: a global analysis. Bull World Health Organ 93: 446-456

2. Ligibel JA, Alfano CM, Hershman DL, Merrill JK, Basen-Engquist K, et al. (2017) American society of clinical oncology summit on addressing obesity through multidisciplinary provider collaboration: key findings and recommendations for action. Obesity (Silver Spring) 25 Suppl 2: S34-S39.

3. Sedov VM, Soloviev MO, Muzhikov SP (2010) Epidemiological characteristics of the metabolic syndrome in the North-West region of Russia. Metabolic syndrome: an interdisciplinary problem. Preventive and clinical medicine 222 3-4.
4. Schauer DP, Feigelson HS, Koebnick C, Caan B, Weinmann S, et al. (2017) Bariatric surgery and the risk of cancer in a large multisite cohort. Ann Surg.

5. Borisenko O, Lukyanov V, Johnsen SP, Funch-Jensen P (2017) Cost analysis of bariatric surgery in Denmark made with a decision-analytic model. Dan Med J 64

6. Sedov VM (2009) Laparoscopic obesity surgery. A practical guide, Atlas, pp. 192.

7. Althuwaini S, Bamehriz F, Aldohayan A, Alshammari W, Alhaidar S, et al (2017) Prevalence and predictors of gastroesophageal reflux disease after laparoscopic sleeve gastrectomy. Obes Surg.

8. DO C (1997) Introduction to pathophysiology of gastroesophageal reflux. Gastroenterol Int 10: 100-110.

9. Chen RY, Burton PR, Ooi GJ, Laurie C, Smith Al, et al. (2017) The physiology and pathophysiology of gastroesophageal reflux in patients with laparoscopic adjustable gastric band. Obes Surg 27: 2434-2443.

10. Slater BJ, Rothenberg SS (2017) Fundoplication. Clin Perinatol 44: 795-803

11. Bailey ME, Garrett WV, Nisar A, Boyle NH, Slater GH (2003) Day-case laparoscopic Nissen fundoplication. Br J Surg 90: 560-562.

12. Puhan MA, Guyatt GH, Armstrong D, Wiklund I, Fallone CA, et al. (2006) Validation of a symptom diary for patients with gastro-oesophageal reflux disease. Aliment Pharmacol Ther 23: 531-541. 